\title{
Does background intensity estimation influence the iterative restoration of microscope images?
}

\author{
Moacir P. Ponti Jr.*, Nelson D. A. Mascarenhas ${ }^{\dagger}$ \\ * Instituto de Ciências Matemáticas e de Computação (ICMC) - Universidade de São Paulo (USP) \\ Caixa Postal 668, 13560-970, São Carlos-SP, Brazil \\ ${ }^{\dagger}$ Departamento de Computação (DC) - Universidade Federal de São Carlos (UFSCar) - São Carlos-SP, Brazil
}

\begin{abstract}
A new background estimation method is proposed to improve iterative restoration of microscope images. The method is based on a sequence of subtractions performed in the beginning of an iterative restoration procedure. A series of experiments using background intensity detection methods were carried out in order to analyse the influence of a correct estimation of the background. The restoration results as a function of the detected background value is discussed. The results indicates a strong influence of the estimate on the restoration of images. When compared to an optimal errorbased method, the proposed method was faster and obtained a good approximation to the correct value.
\end{abstract}

Keywords-image restoration, background estimation, microscopy;

\section{INTRODUCTION}

The acquisition of digital images through devices that detect some physical measure such as light or other radiation, temperature, sound, etc., has limitations that generate an output image that is not equal to the "real" image. Each step of the imaging chain, the lenses, film, detector, digitizer, etc. contributes to the degradation of the image. Therefore every imaging system degrades the image in some way. Image restoration methods are designed to remove or reduce these degradations including: blurring that can be introduced by optical systems, image motion, electronic, thermal or photonic noise, and others [1]. It is possible to characterize the sources of degradation so that the undesired effects can be reduced. When a prior knowledge about the image is available, a model can be developed in order to fit the observed image to a restoration model.

The restoration of images based on additional a priori information about the image and the system is an interesting approach. The use of good prior information makes possible to estimate frequency components of the image that are not passed thought the imaging system [2], and also to reconstruct signals with missing samples or irregularly-sampled spaces [3]. The prior knowledge can be used to impose constraints that the restored image must satisfy. Constrained restoration methods widely used are the constrained least squares method [4], and Richardson-Lucy iterative algorithm [5], [6], that uses the point-spread function of the imaging equipment and the image statistics to obtain restoration through a probabilistic approach [7]. Project onto convex sets (POCS) is another method that can be used to perform constrained restoration [8].

Some common prior information used by algorithms are the: i) image levels are non-negative, ii) image levels are limited, iii) object has finite size, and iv) image is bandlimited. These information are general and can be used in several applications. In addition, another strong constraints can be found by extracting information directly from the image. It can be done for specific images, for example, by defining geometric constraints [9], or also for a set of images, using edge information for the reconstruction of magnetic ressonance spectroscopic data as in [10]. The background information was shown to have an important role in biomedical image restoration and other applications [11]. In this paper the influence of background estimation will be analysed in the context of iterative restoration of microscopy images. A novel method is proposed to obtain an approximation of the optimal value for the background. Existing background estimation methods are studied and the performance is analysed as a function of the background information.

The next section defines the image formation model and an iterative restoration method used in this paper. Section III describes the characteristics of background in microscopy images. Experiments and results are described and discussed in section $\mathrm{V}$ and the concluding remarks in section VII.

\section{ImAGe Formation Model AND ITERATIVE RESTORATION}

Biomedical images are acquired using some imaging system such as microscopes, ultrasound, tomographs and nuclear magnectic ressonance equipments. The image formation is often modeled using the impulse response function of the system. This is an interesting model because the restoration procedure can be posed as an inverse problem. In optical microscopy, the images generally have two sources of noise: an additive and signal-independent noise caused by the effect of the electronic recording system; due to the photon counting nature of light based sensors, there is an another more important source of noise, specially in lowlevel light situations. It is a signal-dependent noise and can 
be well modeled by a Poisson distribution. Considering each value of the observed image $g(x, y, z)$ to be a realization of a random variable described by a Poisson process, the model can be written as:

$$
g(x, y, z)=\mathcal{N}\{f(x, y, z) * h(x, y, z)\},
$$

where $\mathcal{N}\{\}$ represents a random process, more specifically a Poisson process, $g$ is the observed image, $h$ the point spread function (PSF) that models the blurring due to the optical system, $f$ is the original image, $*$ is a convolution operator. This kind of process produces noise that is correlated with the image given by $f(x, y, z) * h(x, y, z)$.

The image formation for many biomedical acquisition systems can be modeled using Eq. 1.

The Richardson-Lucy (R-L) algorithm [5], [6] uses a probabilistic approach to restore images: given a degraded image $g$, what is the image $\hat{f}$ that maximizes the probability of observing the image $g$ ? Considering the image as an observation of a Poisson process, the likelihood function would be:

$$
p(g \mid \hat{f})=\prod_{\mathbf{x}} \frac{h(\mathbf{x}) * \hat{f}(\mathbf{x})^{g(\mathbf{x})} e^{-h(\mathbf{x}) * \hat{f}(\mathbf{x})}}{g(\mathbf{x}) !},
$$

where $\mathbf{x}$ represents $(x, y, z)$ for a 3D signal.

The R-L algorithm minimizes the functional $L(\hat{f})=$ $-\log p(g \mid \hat{f})$, giving the maximum likelihood estimation:

$$
L(\hat{f})=\sum_{\mathbf{x}}-g(\mathbf{x}) \log [(h * \hat{f})(\mathbf{x})]+(h * \hat{f})(\mathbf{x}) .
$$

The R-L iteration is given by:

$$
\hat{f}_{n+1}(\mathbf{x})=\left[\left(\frac{g(\mathbf{x})}{\hat{f}(\mathbf{x}) * h(\mathbf{x})}\right) * h(\mathbf{x})\right] \times \hat{f}_{n}(\mathbf{x}) .
$$

The R-L method is suited to restore images that can be modeled by a Poisson distribution, for example, images acquired under low-light conditions from telescopes and optical microscopes. It is still considered in the literature one of the best solutions to such cases, and that is why the $\mathrm{R}-\mathrm{L}$ method is used in this study to restore the images. An important observation is that R-L has a non-negativity property that was shown to improve restoration of frequencies beyond the microscope resolution.

The use of background information in iterative restoration can be used as a constraint prior to the restoration and possibly improves the quality of the results of iterative restoration methods. The improvement is, however, sensitive to the method used to obtain the background region.

\section{BIOMEDICAL IMAGES AND THE BACKGROUND CHARACTERISTICS}

In fluorescence microscopy, signals not originating from the specimens of interest are also acquired, mainly in the region of background where the fluorophore is also present and emmits light. Images obtained in other biomedical applications, such as: nuclear magnetic ressonance imaging, computerized tomography, ultrasound imaging, microscopy, etc., specially in three-dimensional images, also have a very dense background region. The information that lies on this background region is not useful and it is sometimes removed in some methods before processing the image [11]. The background region in a biomedical image usually corresponds to the majority of pixels (or voxels, in the 3D case).

To observe this characteristic, the histograms of several images were analysed. As demonstrated in previous studies for confocal images [12], the wide-field image histograms are unimodal and the background is concentrated between zero and the mean intensity of the observed intensities result obtained experimentally by van Kempen et. al [11].

\section{BACKGROUND ESTIMATION METHODS}

The biomedical image formation is often modeled using the impulse response function of the system. This is an interesting model since the restoration procedures can be seen as an inverse problem. However, depending on the condition of the sample that is been imaged. it is important to consider the background information present in such images. The models used in most methods attempt to restore objects but often do not consider visual information (light) not originated from the object itself. However, the background knowlegde can be used to improve restoration since it can be extracted before the processing and used as an information for a constrained restoration approach.

\section{A. Maximum of the histogram}

In general, the background can be characterized as being of low intensity and of a low spatial frequency. In images of sparse objects, the majority of the voxels are background voxels, and a histogram-based algorithm can be used to estimate the background.

The maximum of the histogram can be used when the distribution of the main source of noise in the image is unimodal (this assumption holds for Poisson noise). This method uses the maximum value of the histogram to subtract the whole image from this value. Negative voxels are clipped to zero. Figure 1 shows an example of a typical fluorescence microscopic image histogram (a), and the histogram after background removal by the maximum value.

\section{B. Mathematical morphology}

In images of dense objects or in images with a nonconstant background, the histogram-based approach will not be very accurate (still the maximum of the lowest intensity peak might give a reasonable estimate). In these cases an approach based on mathematical morphology might work [11]. An opening operation can be used to estimate the shape of the background. An approach is to use an histogram 


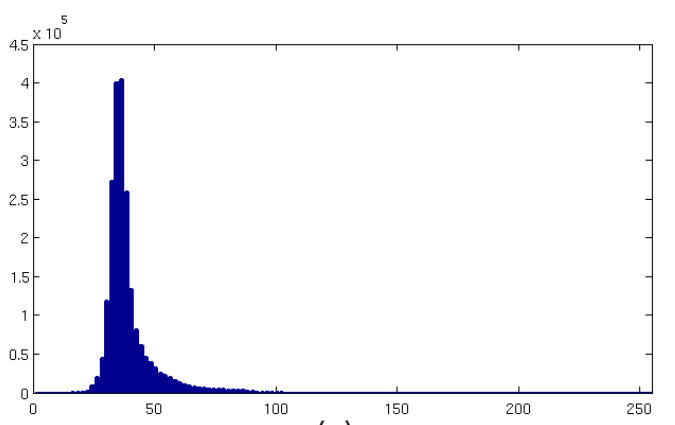

(a)

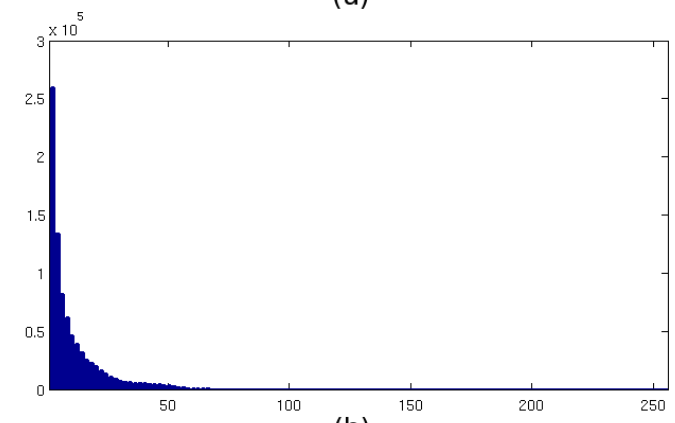

(b)

Figure 1. Example of maximum of histogram subtraction - (a) observed image histogram, (b) background subtracted image histogram

to find the first peak value, $p$, between zero and the mean intensity. The values inside the interval $[0, p]$ are set to zero. Let $g_{b}$ be the background region found by this procedure. An opening morphological operation is carried out over $g_{b}$, resulting in:

$$
g_{m}(\mathbf{x})=\left(g_{b} \ominus d\right)(\mathbf{x}) \oplus d(\mathbf{x}),
$$

where $g_{m}$ is the background region after a closing morphological operation using a disk $d$ of an arbitrary size. The operators $\ominus$ and $\oplus$ represent, respectively, an erosion and a dilation procedures. The region where $g_{m}=0$ is considered to be the new background region. This method prevents the existence of isolated values in background regions to be treated as the object to be processed.

\section{Pre-segmentation}

The accuracy and the bias of the fit will be improved if it is done over background voxels only. Therefore the image needs to be coarsely segmented in object and background voxels. A way of doing this is to use the noise variance as a criterion. Given a first histogram-based estimate of the background intensity, a voxel can be labeled as an object voxel if its intensity is more that $n$ ( 2 or 3 ) times the standard deviation of the noise. This method requires the estimate of noise statistics.

\section{Mean-squared error method}

This approach, proposed by [11], also uses the maximum histogram-based estimate of a value as a first estimation.
After that, the algorithm uses an error based on the difference between the observed image and the restoration result blurred by the microscope's OTF with the added background to measure the performance as a function of the background:

$$
\sum[g(\mathbf{x})-(h(\mathbf{x}) \hat{f}(\mathbf{x})+b(\mathbf{x}))]^{2}
$$

where $\hat{f}$ is the restored image, $b$ is the estimated background. By optimizing this measure as function of background, the optimal background can be determined. It uses the meansquare-error between the acquired image and the restoration result blurred by the microscope's OTF with the added background.

This method obtains the optimal value for the background (to be subtracted) by running a restoration method and computing the error for several values of background. After finding the value that minimizes the error, it is used to subtract the background and, finally, process the image. A disadvantage of this method is that it requires a few restoration results with some restoration method. This could lead to a high computational complexity and, consequently, an unacceptably long processing time [11].

\section{E. Sequence of subtractions method}

In this paper we propose a new method to restore images using a background mean-squared error and a POCS-based iterative restoration. It computes the error in Eq. 6 as iterations are performed. The advantage is that it does not need several restoration tests before performing the definitive restoration. The method can be described as follows:

1) Let $b_{0}$ be the first non-zero value in the image histogram.

2) Perform the subtraction $s_{0}=g-b_{0}$, where $g$ is the observed image.

3) Perform one restoration iteration (in this study we used a R-L iteration) with image $s_{0}$.

4) Obtain the first non-zero value, $b_{i}$, in the current restored image, $\hat{f}_{i}$.

5) Perform an iteration using the the image $s_{i}=\hat{f}_{i}-b_{i}$ and compute the error.

6) If the error increases: rollback to the previous estimate and proceed with the iterative restoration without further subtractions.

7) Else, if the error decreases: use the resulting image as the current estimation and repeat steps 4-6.

8) Otherwise: use the resulting image as the current estimation and proceed with the iterative restoration without further subtractions.

\section{EXPERIMENT DESIGN}

A series of experiments were carried out using the methods described in the previous sections. The restoration method used to test each background detection method was the Richardson-Lucy (R-L) iterative restoration. The images 
used to run the experiments are: a bead synthetic image, with resolution of $128 \times 128 \times 128$, and a real image obtained with a wide-field fluorescence microscope with resolution of $256 \times 256 \times 32$ pixels, both images with 256 gray levels.

The synthetic image was convolved with a theoretical microscope PSF, following the model of Gibson and Lanni [13]. Poisson noise was applied to the image and the background intensity value was set to 7 (seven). Note that the parameter for Poisson noise, $\lambda$, represents the mean number of occurrences and its variance [14]. Therefore, one cannot set multiple noise leves for one image because the noise is related to the intensity pixel value observed. Sections of the synthetic image (original and degraded) are shown in Figure 2 and sections of the real image are shown in Figure 3.
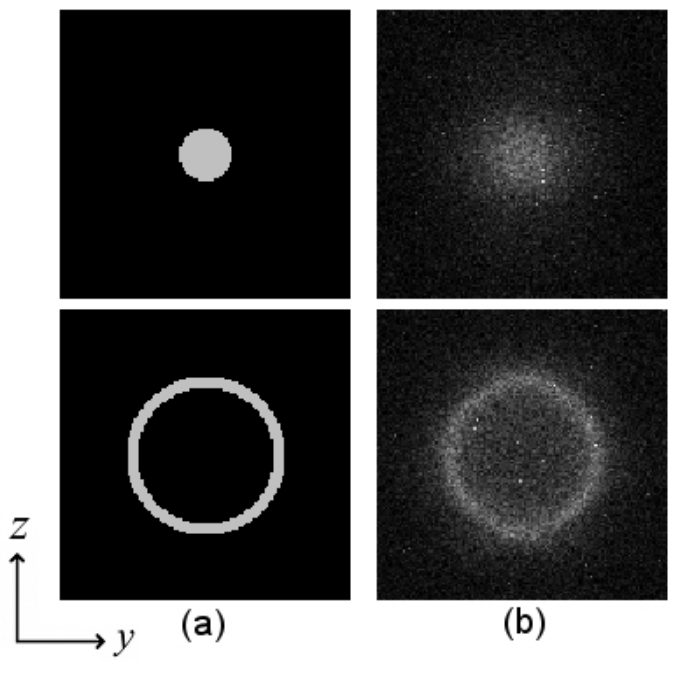

(b)

Figure 2. Synthetic bead image (a) original, (b) degraded, sections: $32, y, z$ (top) and $128, y, z$ (bottom)

Both phantom and real images were restored using the R-L algorithm. Before restoration, the following methods described in section IV were applied to detect the background value: maximum of the histogram (MAXH), mathematical morphology (MM), background mean-squared error (BMSE), and sequence of subtractions (SOS). A restoration was also carried out without using the background detection. The stop criterion used is $\varepsilon \leq 0.001$, where $\varepsilon$ is the residual, that can be defined as:

$$
\varepsilon=\frac{\left\|\hat{f}_{n}-\hat{f}_{n-1}\right\|}{\left\|\hat{f}_{n}\right\|}
$$

To obtain the optimal value using the BMSE, we compute 50 iterations for each value between zero and the mean intensity value of the image, since it was experimentally found that the correct value lie in this interval [15].

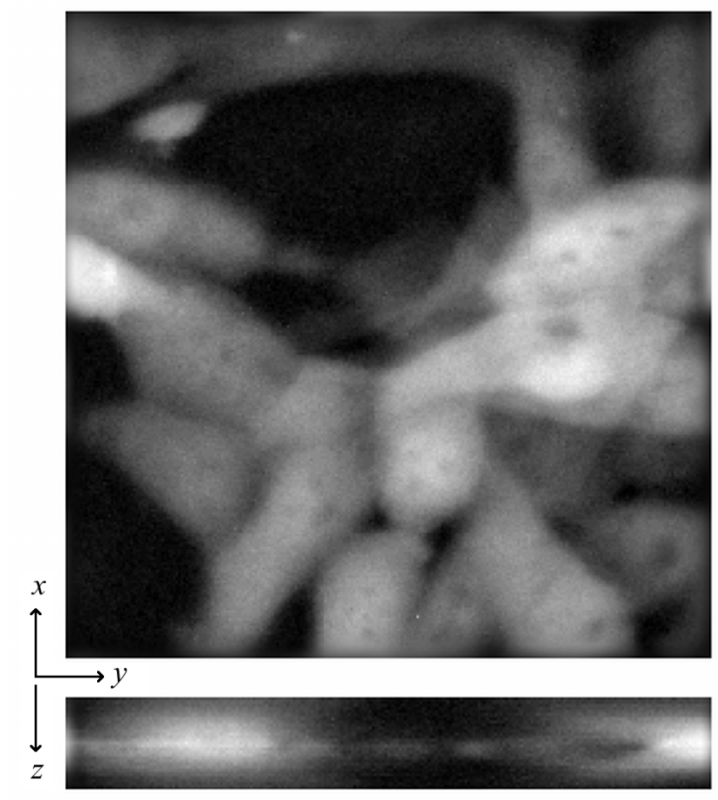

Figure 3. Real image sections: $x, y, 16$ (top) and 100, $y, z$ (bottom)

\section{A. Performance Evaluation}

Three measures are used to evaluate the restoration performance: the improvement on signal-to-noise ratio (ISNR), the universal image quality index (UIQI), and the practical image bandpass to observe the presence of new higher frequencies on the restored image.

The ISNR is computed using the following equation:

$$
\operatorname{ISNR}=10 \log _{10}\left(\frac{\sum_{i}\left\|g\left(\mathbf{x}_{i}\right)-f\left(\mathbf{x}_{i}\right)\right\|}{\sum_{i}\left\|\hat{f}\left(\mathbf{x}_{i}\right)-f\left(\mathbf{x}_{i}\right)\right\|}\right),
$$

where $g(\mathbf{x})$ is the degraded image, $f(\mathbf{x})$ the original image and $\hat{f}(\mathbf{x})$ the restored image. The ISNR compares the degraded and restored images with the original, and yields a number that measures the relative improvement.

The UIQI represents an attempt to measure the structural distortion, instead of an error, and is defined as:

$$
\mathrm{UIQI}=\frac{4 \sigma_{x y} \cdot \bar{x} \bar{y}}{\left(\sigma_{x}^{2}+\sigma_{y}^{2}\right) \cdot\left(\bar{x}^{2}+\bar{y}^{2}\right)}
$$

where $x$ and $y$ are the original and processed images. The letters with bars are averages, $\sigma^{2}$ the variance of the images, and $\sigma_{x y}$ the correlation between $x$ and $y$. The dynamic range of UIQI is $[-1,1]$. The value, 1 , is achieved only if $x=y$.

To assess the restoration beyond the theoretical microscope resolution, we use a method described by Conchello [7], the practical bandpass of the image, as the region of the frequency domain where the absolute value is larger than $1 \%$ of its peak value. That is, the coefficients where:

$$
|\hat{F}(u, v, w) / \hat{F}(\mathbf{0})|>0.01
$$




\section{Results}

In this section we present results from a simulation experiment in which the performance of image restoration algorithms is measured as a function of the estimated background. The improvement in signal-to-noise ratio (ISNR) is computed. The universal image quality index (UIQI) to measure structural and illumination distortions is also evaluated. The practical bandpass is used to gives an insight on what extent the algorithms restore information beyond the microscope resolution.

The results showed, as expected, a strong influence of the correct background estimation on the restoration of images. As shown in Table I, incorrect estimates obtained by MAXH and $\mathrm{MM}$ methods caused a decrease in the performance even when compared with results achieved without background estimation. The visual inspection of bead image (Fig. $4 \mathrm{c}$ and d) shows artifacts inside the bead, while the BMSE and SOS methods preserved better the object shape. Although the MAXH and MM methods can be suited to some problems, one must be use them carefully in order to not deteriorate the results.

The BMSE method found the correct value (7) for the synthetic image and the expected value for the real image (4) (Table II), while the MAXH and MM methods overestimated the background in both cases. The proposed method was not able to find exactly the optimal value but a good approximation of it, yielding a relatively good result for the restoration. It must be observed that, for the synthetic image, the BMSE method used 600 iterations to obtain the optimal value, and for the real image, 450 iterations.

The restoration results for real images can only be assessed visually, showing in this experiment better restoration specially through the $z$ axis (Fig.5 e and $\mathrm{f}$ ), presenting less spreadness in this direction.

A good estimation of the background value can help the restoration algoritm to restore images faster and to be more stable. Figure 6 shows the ISNR increasing faster for methods that uses better approximations of the background value. It also shows that, for a high-level noise - as in the images used in this paper -, a correct knowledge about background can help the algorithm not to rapidly diverge.

The overestimation of the background, which removes object information, can severely affect performance as shown in Figure 7. The UIQI quickly drops from 0.71 to 0.53 with a difference of just two intensity values. The underestimation of background value was not so critical, as can also be seen in Figure 7. This influence on non-linear image restoration was demonstrated before by van Kempen et al. [11]. However, the method proposed by these authors needed several additional iterations to obtain the optimal value, while the proposed method finds an approximation as the iterations are performed, reducing the computational complexity.

All background detection methods enhanced better the high frequencies, when compared with the non-linear restoration method alone (R-L). This improvement on the restoration of frequencies beyond the microscope resolution occurs because the background subtraction works as a finite-support constraint on the space domain of image. As demonstrated before [16], [8], this constraint can improve restoration of components in frequency domain.

Table I

SYNTHETIC BEAD IMAGE RESULTS: ISNR, UIQI, PRACTICAL BANDPASS (PB), TOTAL NUMBER OF ITERATIONS (IT), AND BACKGROUND VALUE (BVAL)- ESTIMATED (EST) AND ACTUAL (ACT).

\begin{tabular}{l|rrrrr}
\hline Methods / Results & ISNR & UIQI & PB & It & BVal Est(Act) \\
\hline \hline R-L & 4.29 & 0.59 & 2647 & 148 & $0(7)$ \\
MAXH + R-L & 3.56 & 0.47 & 13007 & 120 & $10(7)$ \\
MM + R-L & 3.89 & 0.53 & 11351 & 126 & $9(7)$ \\
BMSE + R-L & 5.28 & 0.70 & 10729 & $161 *$ & $7(7)$ \\
SOS + R-L & 5.17 & 0.71 & 8075 & 183 & $6(7)$ \\
\hline * - plus 600 iterations to compute optimal background value
\end{tabular}

Table II

REAL IMAGE RESULTS: PRACTICAL BANDPASS (PB), TOTAL NUMBER OF ITERATIONS (IT) AND ESTIMATED BACKGROUND VALUE (BVAL).

\begin{tabular}{l|rrr}
\hline Methods / Results & PB & It & BVal \\
\hline \hline R-L & 711 & 98 & 0 \\
MAXH + R-L & 1317 & 140 & 8 \\
MM + R-L & 1179 & 115 & 8 \\
BMSE + R-L & 893 & $103^{*}$ & 4 \\
SOS + R-L & 897 & 105 & 5 \\
\hline * - plus 450 iterations to compute optimal background value
\end{tabular}

\section{CONCLUSION}

A new method to obtain background estimation with the use of an iterative restoration method was proposed showing good approximations of the optimal value and good numerical results for ISNR and UIQI. A strong influence of a correct background intensity detection was observed; the overestimation of this value can easily deteriorate the restoration of images, causing the algorithm to diverge faster, specially in presence of noise. The competing method, although optimal, require several additional iterations to converge. The proposed method was able to find a good approximation for the optimal value while the first iterations were performed in the restoration process, without hampering the restoration itself. New efforts can be directed in order to prevent the overestimation of intensity values. In nuclear magnetic ressonance and computerized tomography, the background issue is not as complex as it is in optical microscopy. However, methods developed to improve microscope image condition can be very useful with other biomedical image acquisition systems. 


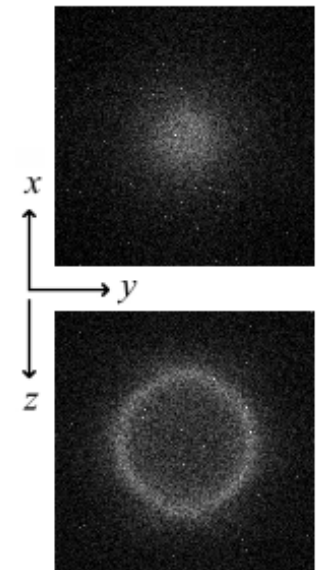

(a)
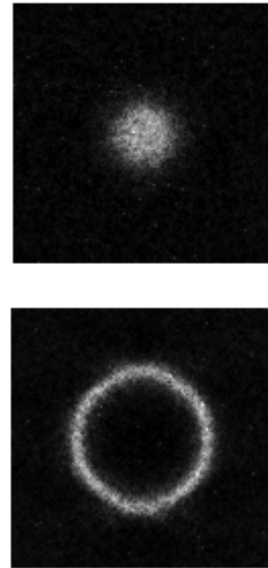

(b)
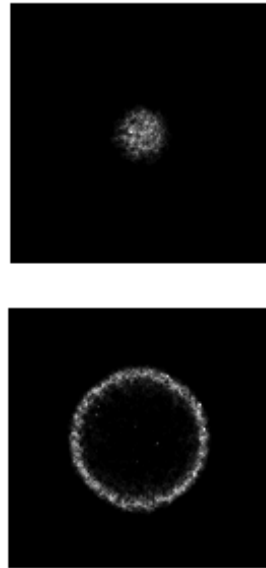

(c)
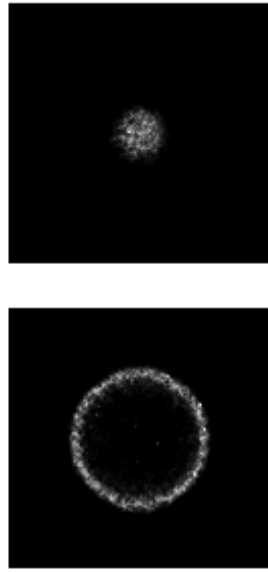

(d)
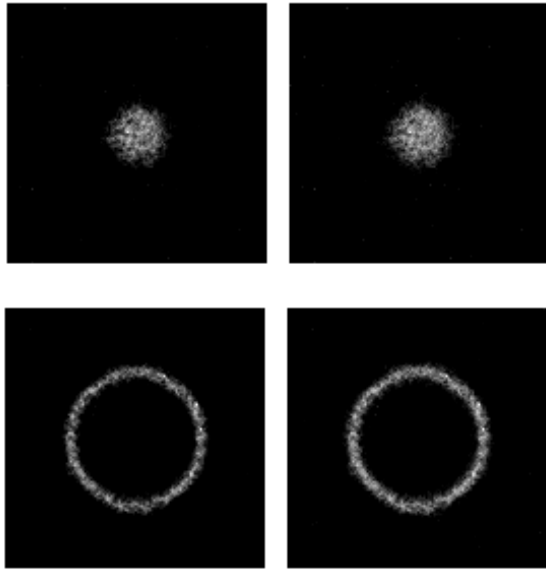

(e)

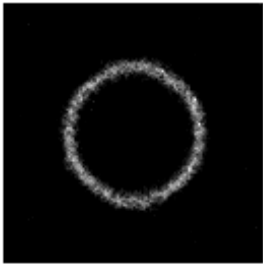

(f)

Figure 4. Results for the synthetic bead image: (a) observed, and restored using: (b) R-L, (c) MAXH + R-L, (d) MM + RL, (e) BMSE + RL, (f) SOS + R-L

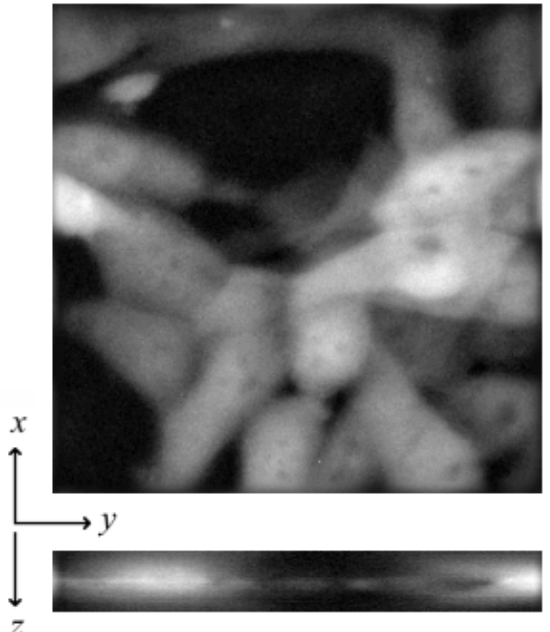

(a)

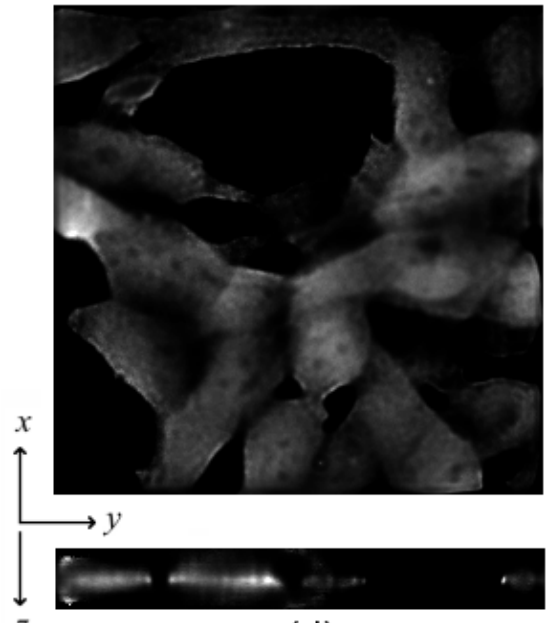

(d)

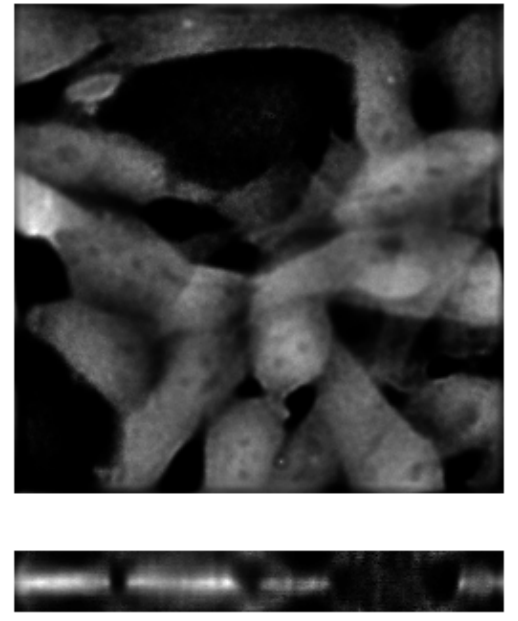

(b)

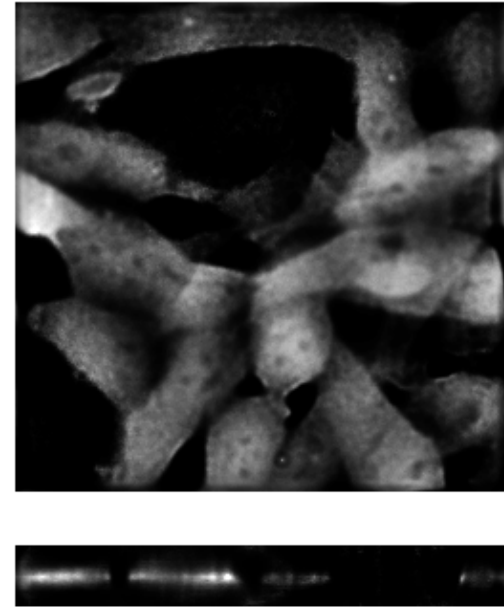

(e)

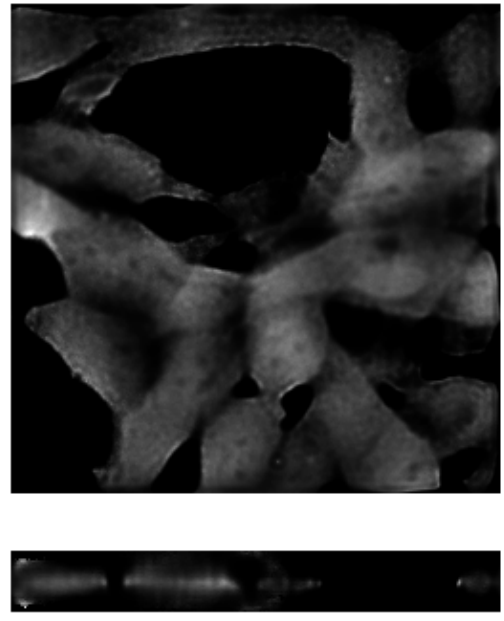

(c)

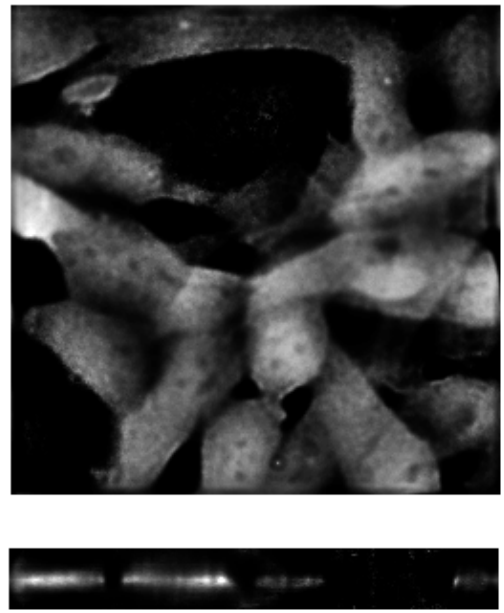

(f)

Figure 5. Results for the real image: (a) observed, and restored using: (b) R-L, (c) MAXH + R-L, (d) MM + RL, (e) BMSE + RL, (f) SOS + R-L 


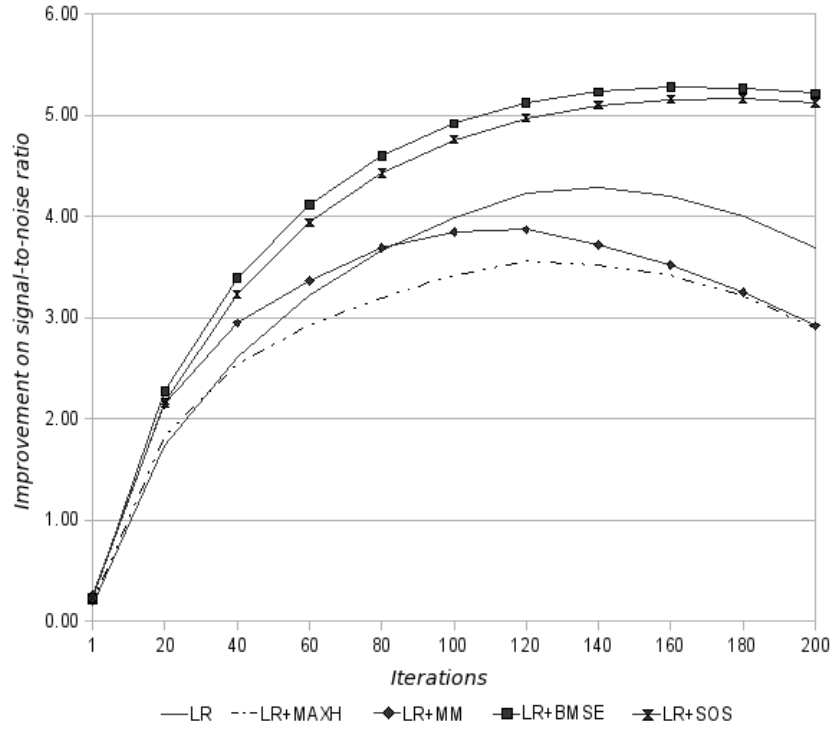

Figure 6. ISNR measure as a function of the restoration iteration

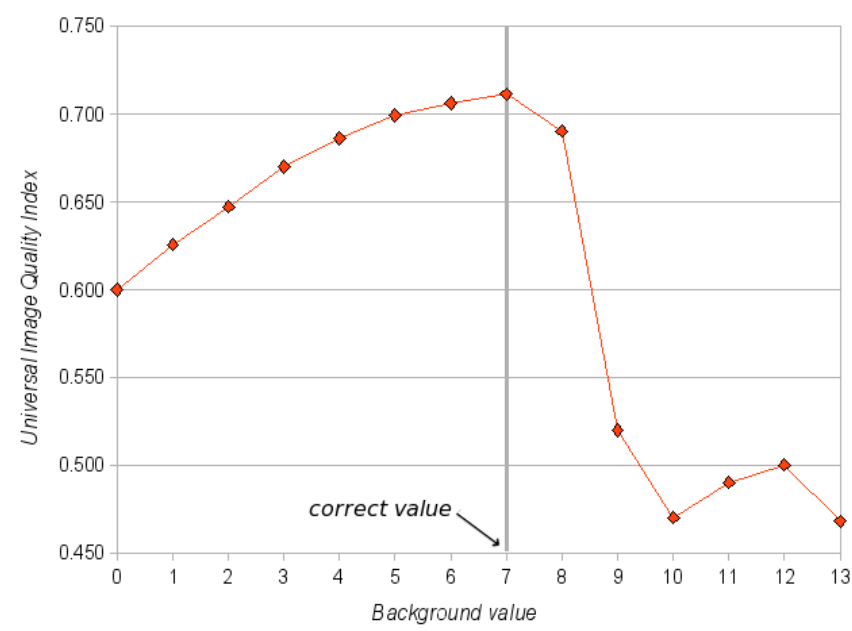

Figure 7. Restoration performance (UIQI) as a function of the background estimation with 7 (seven) as the actual background value

\section{ACKNOWLEDGMENT}

The authors would like to thank Fundação Hemocentro de Ribeirão Preto for providing the microscope images. This study was also partially supported by a CAPES scolarship.

\section{REFERENCES}

[1] K. R. Castleman, Digital Image Processing. New York, USA: Prentice-Hall, 1996.

[2] J.-A. Conchello and J. W. Lichtman, "Optical sectioning microscopy," Nature Methods, vol. 2, no. 12, pp. 920-931, 2005.
[3] R. Stasiski and J. Konrad, "POCS-based image reconstruction from irregularly-spaced samples," in Proc. IEEE Int. Conf. Image Processing (ICIP'O0), vol. 2, Vancouver, BC, Canada, 2000, pp. 315-318.

[4] W. Carrington, R. Lynch, E. Moore, G. Isenberg, K. Fogarty, and F. Fay, "Superresolution three-dimensional images of fluorescence in cells with minimal light exposure," Science, vol. 268, pp. 1483-1487, 1995.

[5] W. Richardson, "Bayesian-based iterative method of image restoration," J. Opt. Soc. Am., vol. 62, no. 1, pp. 55-59, 1972.

[6] L. Lucy, "An iterative technique for the rectification of observed distributions," The Astronomical Journal, vol. 79, no. 6, pp. 745-765, 1974.

[7] J.-A. Conchello, "Superresolution and convergence properties of the expectation-maximization algorithm for maximumlikelihood deconvolution of incoherent images," J. Opt. Soc. Am. A, vol. 15, no. 10, pp. 2609-2619, 1998.

[8] H. Stark and Y. Yang, Space Vector Projections. Wiley, 1998.

[9] G. Danuser, "Super-resolution microscopy using normal flow decoding and geometric constraints," J. Microscopy, vol. 204, no. 2, pp. 136-149, 2001.

[10] T. Denney-Jr. and S. J. Reeves, "Bayesian image reconstruction from fourier-domain samples using prior edge information," J. Elec. Imaging, vol. 14, no. 4, p. 043009, 2005.

[11] G. van Kempen and L. van Vliet, "Background estimation in nonlinear image restoration," J. Opt. Soc. Am. A, vol. 17, no. 3 , pp. $425-433,2000$.

[12] N. Dey, L. Blanc-Feraud, C. Zimmer, P. Roux, Z. Kam, J.C. Olivo-Marin, and J. Zerubia, "Richardson-Lucy algorithm with Total Variation regularization for 3D confocal microscope deconvolution," Microscopy Research and Technique, vol. 69, no. 4, pp. 260-266, 2006.

[13] F. Gibson and F. Lanni, "Experimental test of an analytical model of aberration in an oil-immersion objective lens used in three-dimensional light microscopy," J. Opt. Soc. Am. A, vol. 8 , no. 11 , pp. 1601-1613, 1991 .

[14] D. Snyder and M. Miller, Random Point Processes in Time and Space. Springer Verlag, 1991.

[15] N. Dey, L. Blanc-F'eraud, C. Zimmer, P. Roux, Z. Kam, J.-C. Olivo-Marin, and J. Zerubia, "3D microscopy deconvolution using Richardson-Lucy algorithm with Total Variation regularization," Institut National de Recherche en Informatique et en Automatique, Paris, France, Tech.Report. n.5272, 2004.

[16] B. Hunt, "Super-resolution of imagery: understanding the basis for recovery of spatial frequencies beyond the diffraction limit," in Information, Decision and Control, 1999. IDC 99. Proceedings. IEEE, 1999, pp. 243-248. 